Advanced Nonlinear Studies 3 (2003), 289-299

\title{
Perturbation of Global Solution Curves for Semilinear Problems
}

\author{
Philip Korman * \\ Department of Mathematical Sciences \\ University of Cincinnati, Cincinnati, Ohio 45221-0025 \\ e-mail: kormanp@math.uc.edu \\ Yi Li \\ Department of Mathematics \\ University of Iowa, Iowa City, Iowa 52242 \\ e-mail:yi-li@uiowa.edu \\ Tiancheng Ouyang \\ Department of Mathematics \\ Brigham Young University, Provo, Utah 84602 \\ e-mail: ouyang@math.byu.edu \\ Received in revised form 4 April 2003 \\ Communicated by Norman Dancer
}

\begin{abstract}
We revisit the question of exact multiplicity of positive solutions for a class of Dirichlet problems for cubic-like nonlinearities, which we studied in [6]. Instead of computing the direction of bifurcation as we did in [6], we use an indirect approach, and study the evolution of turning points. We give conditions under which the critical (turning) points continue on smooth curves, which allows us to reduce the problem to the easier case of $f(0)=0$. We show that the smallest root of $f(u)$ does not have to be restricted.
\end{abstract}

*Supported in part by the Taft Faculty Grant at the University of Cincinnati 
1991 Mathematics Subject Classification. 34B15.

Key words. Exact number of solutions, perturbation of critical points

\section{Introduction}

In this paper we revisit the question of exact multiplicity of positive solutions for a class of cubic-like nonlinearities. Our prototype is the Dirichlet problem (for $u=u(x))$

$$
u^{\prime \prime}+\lambda(u-a)(b-u)(c-u)=0 \quad x \in(-1,1), \quad u(-1)=u(1)=0
$$

with constants $0 \leq a<b<c$, and a positive parameter $\lambda$. Observe that positive solutions of (1.1) are even functions, with the maximum value of $u(0)$, see [4]. This problem was originally studied using the time-maps, see J. Smoller and A. Wasserman [8] and S.-H. Wang [10], [11]. While the time-mapping method is straighforward, its application to the problem (1.1) is rather involved. In [6] the present authors have used bifurcation theory approach, involving the Crandall-Rabinowitz theorem [2], and computation of the direction of bifurcation. Let us briefly review the results of both approaches for $f(u)=(u-a)(b-u)(c-u)$ and similar nonlinearities. The case $a=0$ turned out to be relatively easy for both approaches. A necessary condion for the existence of positive solutions is then $c>2 b$ (which says that the area of the positive hump of $f(u)$ is greater than that of the negative one). In this case there is a critical $\lambda_{0}$, so that the problem has exactly zero, one or two positive solutions, depending on whether $\lambda<\lambda_{0}, \lambda=\lambda_{0}$ or $\lambda>\lambda_{0}$ (and more details are available on the solution curve, see [6]). If $a>0$ it is easy to show that there is a monotone in $\lambda$ curve of positive solutions, satisfying $u(x)<a$, the lower curve. The necessary condition for the existence of additional positive solutions is now $c>2 b-a$, as can be easily seen by considering $g(u) \equiv f(u+a)$, with $g(0)=0$. Then for $a$ not too large the problem (1.1) has a parabola-like curve of solutions, similar to the one from $a=0$ case. (All solutions on this curve satisfy $u(0)>a$. Hence we refer to it as the upper curve.) The restriction on $a$ appeared in both [10] and [6], although the exact conditions were different. In the present paper, among other things, we observe that $a$ does not have to be bounded, if the area of the negative hump of $f(u)$ is almost the same as the area of the positive hump that follows it, i.e. when $c \simeq 2 b-a$.

Instead of computing the direction of bifurcation as we did in [6], we use an indirect approach, and study the evolution of turning points, as the value of $a$ changes. We give conditions under which the critical (turning) points of (1.1) continue on smooth curves. Since at $a=0$ there is only one turning point, the same must be true at other $a$, proving that the upper curve is a parabola-like curve. In case the positive and negative humps of $f(u)$ are not nearly balanced, as mentioned above, we still need to restrict $a$, and the condition turned out to be the same as in our earlier paper [6], even though the approach is different. The present approach appears to be more straightforward, and more likely to extend to other situations. 
We recall next the Crandall-Rabinowitz theorem [2], which will be used in the proof.

Theorem 1.1 [2] Let $X$ and $Y$ be Banach spaces. Let $(\bar{\lambda}, \bar{x}) \in \mathbf{R} \times X$ and let $F$ be a continuously differentiable mapping of an open neighborhood of $(\bar{\lambda}, \bar{x})$ into $Y$. Let the null-space $N\left(F_{x}(\bar{\lambda}, \bar{x})\right)=\operatorname{span}\left\{x_{0}\right\}$ be one-dimensional and codim $R\left(F_{x}(\bar{\lambda}, \bar{x})\right)=1$. Let $F_{\lambda}(\bar{\lambda}, \bar{x}) \notin R\left(F_{x}(\bar{\lambda}, \bar{x})\right)$. If $Z$ is a complement of $\operatorname{span}\left\{x_{0}\right\}$ in $X$, then the solutions of $F(\lambda, x)=F(\bar{\lambda}, \bar{x})$ near $(\bar{\lambda}, \bar{x})$ form a curve $(\lambda(s), x(s))=(\bar{\lambda}+\tau(s), \bar{x}+$ $\left.s x_{0}+z(s)\right)$, where $s \rightarrow(\tau(s), z(s)) \in \mathbf{R} \times Z$ is a continuously differentiable function near $s=0$ and $\tau(0)=\tau^{\prime}(0)=0, z(0)=z^{\prime}(0)=0$.

\section{Perturbation and degeneracy of critical points}

We consider positive solutions of the Dirichlet problem

$$
u^{\prime \prime}+\lambda f(u, \epsilon)=0 \quad x \in(-1,1), \quad u(-1)=u(1)=0
$$

depending on positive parameters $\lambda$ and $\epsilon$. We assume that the function $f(u, \epsilon)$ is twice continuously differentiable in $u$ and continuous in $\epsilon$. We will be particularly interested in the critical points of $(2.1)$, i.e. the solution triples $(\lambda, u, \epsilon)$ for which the corresponding linearized problem

$$
w^{\prime \prime}+\lambda f_{u}(u, \epsilon) w=0 \quad x \in(-1,1), \quad w(-1)=w(1)=0
$$

has a nontrivial solution $w(x)$. We recall that any positive solution of (2.1) is an even function, with $u^{\prime}(x)<0$ for $x>0$, and that any nontrivial solution of $(2.2)$ is a positive even function, see [6]. We also recall the following lemma that we proved in $[6]$.

Lemma 2.1 Let $u(x)$ be a critical solution of (2.1), and $w(x)>0$ a corresponding solution of (2.2). Then we have

$$
\int_{-1}^{1} f(u, \epsilon) w d x=\frac{1}{2 \lambda} u^{\prime}(1) w^{\prime}(1)>0 .
$$

We now wish to continue the critical points, when the secondary parameter $\epsilon$ is varied. The following lemma was first proved by E.N. Dancer [3] (see also J. Shi [9]). We present a simpler proof for completeness.

Lemma 2.2 Let $\left(\lambda_{0}, u_{0}, \epsilon_{0}\right)$ be a critical point of (2.1), with $w_{0}$ the corresponding non-trivial solution of (2.2), and assume that

$$
\int_{-1}^{1} f_{u u}\left(u_{0}, \epsilon_{0}\right) w_{0}^{3} d x \neq 0 .
$$

Then there is a unique critical solution $(\lambda(\epsilon), u(\epsilon), \epsilon)$ near $\left(\lambda_{0}, u_{0}, \epsilon_{0}\right)$. 
Proof. We can normalize the solution of $(2.2)$, so that

$$
\frac{1}{2} \int_{-1}^{1} w^{2} d x=1
$$

The equations (2.1), (2.2) and (2.5) give us three equations with four variables, $u$, $w, \lambda$ and $\epsilon$. We show that the implicit function theorem applies. Indeed, we define a map $H(u, w, \lambda, \epsilon): H^{2}(-1,1) \times H^{2}(-1,1) \times R \times R \rightarrow L^{2}(-1,1) \times L^{2}(-1,1) \times R$ as a vector whose entries are the left hand sides of the above equations:

$$
H(u, w, \lambda, \epsilon)=\left[\begin{array}{c}
u^{\prime \prime}+\lambda f(u, \epsilon) \\
w^{\prime \prime}+\lambda f_{u}(u, \epsilon) w \\
\frac{1}{2} \int_{-1}^{1} w^{2} d x
\end{array}\right]
$$

The linearized operator with respect to the first three variables is

$$
\begin{aligned}
& H_{(u, w, \lambda)}^{\prime}\left(u_{0}, w_{0}, \lambda_{0}, \epsilon_{0}\right)\left[\begin{array}{l}
v \\
\theta \\
\tau
\end{array}\right] \\
& =\left[\begin{array}{c}
v^{\prime \prime}+\lambda_{0} f_{u}\left(u_{0}, \epsilon_{0}\right) v+\tau f\left(u_{0}, \epsilon_{0}\right) \\
\theta^{\prime \prime}+\lambda_{0} f_{u}\left(u_{0}, \epsilon_{0}\right) \theta+\lambda_{0} f_{u u}\left(u_{0}, \epsilon_{0}\right) w_{0} v+\tau f_{u}\left(u_{0}, \epsilon_{0}\right) w_{0} \\
\int_{-1}^{1} w_{0} \theta d x
\end{array}\right] .
\end{aligned}
$$

We need to show that this operator is injective and surjective. To see that it is injective, we need to show that the system

$$
\begin{gathered}
v^{\prime \prime}+\lambda_{0} f_{u}\left(u_{0}, \epsilon_{0}\right) v+\tau f\left(u_{0}, \epsilon_{0}\right)=0 \\
\theta^{\prime \prime}+\lambda_{0} f_{u}\left(u_{0}, \epsilon_{0}\right) \theta+\lambda_{0} f_{u u}\left(u_{0}, \epsilon_{0}\right) w_{0} v+\tau f_{u}\left(u_{0}, \epsilon_{0}\right) w_{0}=0 \\
\int_{-1}^{1} w_{0} \theta d x=0
\end{gathered}
$$

has only the trivial solution $(v, \theta, \tau)=(0,0,0)$. The first equation in $(2.8)$ can be regarded as a linear equation for $v$, with a kernel spanned by $w_{0}$, and the right hand side equal to $-\tau f\left(u_{0}, \epsilon_{0}\right)$. Since by Lemma $2.1 f\left(u_{0}, \epsilon_{0}\right)$ is not orthogonal to the kernel, it follows that the first equation is solvable only if $\tau=0$. We then have $v=k w_{0}$, with a constant $k$. We now regard the second equation in (2.8) as a linear equation for $\theta$ with the same kernel, and the right hand side equal to $-k \lambda_{0} f_{u u}\left(u_{0}, \epsilon_{0}\right) w_{0}^{2}$. By our condition (2.4), the second equation is solvable only if $k=0$. We then have $\theta=l w_{0}$, with a constant $l$. ¿From the third equation in (2.8) we conclude that $l=0$, completing the proof of injectivity.

Turning to the surjectivity, we need to show that for any $L^{2}$ functions $a(x)$ and $b(x)$, and for any constant $c$ the problem

$$
\begin{gathered}
v^{\prime \prime}+\lambda_{0} f_{u}\left(u_{0}, \epsilon_{0}\right) v+\tau f\left(u_{0}, \epsilon_{0}\right)=a(x) \\
\theta^{\prime \prime}+\lambda_{0} f_{u}\left(u_{0}, \epsilon_{0}\right) \theta+\lambda_{0} f_{u u}\left(u_{0}, \epsilon_{0}\right) w_{0} v+\tau f_{u}\left(u_{0}, \epsilon_{0}\right) w_{0}=b(x) \\
\int_{-1}^{1} w_{0} \theta d x=c
\end{gathered}
$$


is solvable. Proceeding similarly to the above, we regard the first equation in (2.9) as a linear equation for $v$ with the right hand side equal to $a(x)-\tau f\left(u_{0}, \epsilon_{0}\right)$. By Lemma 2.1 we can choose $\tau$ so that this function is orthogonal to $w_{0}$. Then the first equation in (2.9) has infinitely many solutions of the form $\bar{v}+k w_{0}$, where $\bar{v}$ is any fixed solution, and $k$ any constant. We now turn to the second equation in (2.9), where $\tau$ has been just fixed above. In view of our condition (2.4) we can fix $k$ so that this equation is solvable. We then have $\theta=\bar{\theta}+l w_{0}$, where $\bar{\theta}$ is a fixed solution and $l$ is any number. Finally, from the third equation in (2.9) we uniquely determine $l$.

We say that a critical point $\left(\lambda_{0}, u_{0}, \epsilon_{0}\right)$ is non-degenerate, if the condition (2.4) is satisfied. Next we give some conditions on $f(u)$, which imply the non-degeneracy condition (2.4). We assume that the secondary parameter $\epsilon$ in (2.1) is fixed (or not present) and suppress it in our notations.

Lemma 2.3 Assume that for the function $f(u) \in C^{2}\left(\bar{R}_{+}\right)$there is an $\alpha>0$, so that

$$
f^{\prime \prime}(u)>0 \text { for } 0 \leq u<\alpha, f^{\prime \prime}(u)<0 \text { for } u>\alpha .
$$

Let $u(x)$ be a singular solution of (2.1), such that

$$
\int_{0}^{1} f^{\prime \prime}(u) w^{2} u_{x} d x>0 .
$$

Then the condition (2.4) holds.

Proof. We may assume that

$$
f^{\prime \prime}(u(0))<0,
$$

since otherwise the lemma follows immediately. Near the critical solution differentiate the equation (2.1) twice with respect to the parameter $s$ from the CrandallRabinowitz Theorem 1.1

$$
\begin{gathered}
u_{s s}^{\prime \prime}+\lambda f^{\prime}(u) u_{s s}+2 \lambda^{\prime} f^{\prime}(u) u_{s}+\lambda f^{\prime \prime}(u) u_{s}^{2}+\lambda^{\prime \prime} f(u)=0 \\
u_{s s}(-1)=u_{s s}(1)=0
\end{gathered}
$$

We now set $s=0$. In view of the Crandall-Rabinowitz theorem we have $\lambda^{\prime}(0)=0$, $\left.u_{s}\right|_{s=0}=w$. We then have from (2.13)

$$
\begin{gathered}
u_{s s}^{\prime \prime}+\lambda f^{\prime}(u) u_{s s}+\lambda f^{\prime \prime}(u) w^{2}+\lambda^{\prime \prime}(0) f(u)=0 \\
u_{s s}(-1)=u_{s s}(1)=0 .
\end{gathered}
$$

Combining this problem with the linearized problem (2.2), we express

$$
\lambda^{\prime \prime}(0)=-\frac{\lambda \int_{-1}^{1} f^{\prime \prime}(u) w^{3} d x}{\int_{-1}^{1} f(u) w d x} .
$$


Assuming the lemma to be false, i.e.

$$
\int_{-1}^{1} f^{\prime \prime}(u) w^{3} d x=0
$$

we see from (2.15) that $\lambda^{\prime \prime}(0)=0$. Denoting $u_{s s}=\theta$, we then rewrite (2.14) as

$$
\theta^{\prime \prime}+\lambda f^{\prime}(u) \theta+\lambda f^{\prime \prime}(u) w^{2}=0, \quad \theta(-1)=\theta(1)=0 .
$$

This is a linear problem for $\theta$, with kernel $w$. It has infinitely many solutions of the form $\theta=\theta_{0}+k w$, where $\theta_{0}$ is any fixed solution, and $k$ any constant. By fixing $k$, we may assume that $\theta^{\prime}(1)=0$. We claim that $\theta(x)<0$ near $x=1$. Indeed, since $f^{\prime \prime}(0)>0, \theta(x)$ is a supersolution of the linearized equation (2.2) near $x=1$, and hence it cannot touch the trivial solution of the same equation from above.

Next we show that

$$
\theta(x)<0 \text { for all } x \in[0,1) .
$$

Assume that on the contrary $\theta(\xi)=0$ for some $\xi \in(0,1)$. Multiplying the equation (2.17) by $w$, the equation (2.2) by $\theta$, subtracting the results, and then integrating over $(\xi, 1)$, we have

$$
-w(\xi) \theta^{\prime}(\xi)+\lambda \int_{\xi}^{1} f^{\prime \prime}(u) w^{3} d x=0 .
$$

The first term on the left is clearly non-negative. The second one is strictly positive, in view of (2.16), (2.12), and of our assumption that $f^{\prime \prime}(u)$ changes concavity exactly once. The resulting contradiction proves the claim (2.18), and in particular we see that $\theta(0)<0$. Differentiate the equation (2.1),

$$
u_{x}^{\prime \prime}+\lambda f^{\prime}(u) u_{x}=0 .
$$

Multiplying the equation (2.17) by $u_{x}$, the equation (2.19) by $\theta$, subtracting the results, and then integrating over $(0,1)$, we have

$$
\theta(0) u^{\prime \prime}(0)+\lambda \int_{0}^{1} f^{\prime \prime}(u) w^{2} u^{\prime} d x=0
$$

The first term on the left is positive by (2.18), and the second one is positive because of our assumption (2.11). We have a contradiction, which finishes the proof.

Next we assume that $f(u)$ has three positive roots at $0<a<b<c$, and

$$
\begin{gathered}
f(u)>0 \text { on }[0, a) \cup(b, c), f(u)<0 \text { on }(a, b) \cup(c, \infty), \\
\int_{a}^{c} f(u) d u>0,
\end{gathered}
$$

and it satisfies (2.10) with some $\alpha \in(a, c)$. Let $\beta \in(b, c)$ be the unique point satisfying

$$
f^{\prime}(\beta)=\frac{f(\beta)}{\beta-a} .
$$

(I.e. the point where the straight line $y=u-a$ is tangent to the graph of $y=f(u)$.) The following lemma shows that no turns of the solution curve are possible until the maximum value of the solution reaches a certain level. 
Lemma 2.4 Assume $f(u) \in C^{2}$ satisfies the conditions (2.10) and (2.20). If $u(x)$ is a critical solution of (2.1) then

$$
u(0)>\beta .
$$

Proof. Since $f^{\prime}(u)<0$ for $u<a$, it is easy to see that $u(0)>a$. Let $\xi \in(0,1)$ be the point where $u(\xi)=a$. Denote $z(x)=u(x)-a$. Then

$$
z^{\prime \prime}+f(z+a)=0, \quad z^{\prime}(0)=0, \quad z(\xi)=0 .
$$

If we now assume the lemma to be false, i.e $u(0) \leq \beta$, then

$$
z(0) \leq \beta-a .
$$

We claim that

$$
f^{\prime}(z+a)>\frac{f(z+a)}{z} \text { for } z \in(0, \beta-a) .
$$

Indeed, denoting $g(z)=z f^{\prime}(z+a)-f(z+a)$, we see that $g(0)=g(\beta-a)=0$, while $g^{\prime}(z)=z f^{\prime \prime}(z+a)$ changes sign exactly once on $(0, \beta-a)$, with $g^{\prime}>0$ near $z=0$, and $g^{\prime}<0$ near $z=\beta-a$. It follows that $g(z)>0$ on $(0, \beta-a)$, justifying (2.24). Rewriting the linearized equation as

$$
w^{\prime \prime}+f^{\prime}(z+a) w=0,
$$

and comparing it to (2.23), we see (in view of (2.24)) that by Sturm comparison theorem $w(x)$ must vanish on $(0, \xi)$, which is a contradiction, since $w(x)>0$.

We define a constant $\tau \in(b, c)$ by $f^{\prime}(\tau)=0$. We also define a function $I(u)=$ $f^{2}(u)-2 F(u) f^{\prime}(u)$, where as usual $F(u)=\int_{0}^{u} f(t) d t$.

Lemma 2.5 Assume that $f(u) \in C^{2}$ satisfies the conditions (2.10) and (2.20). Assume that either

$$
\int_{a}^{\tau} f(u) \leq 0
$$

or

$$
I(\beta) \geq 0 .
$$

Then the condition (2.11) holds, and hence any critical point of (2.1) is nondegenerate.

Proof. Since by the previous lemma $u(0)>\beta$, it follows that $f^{\prime \prime}(u(0))<0$. Hence we only need to verify the condition (2.11), which will allow us to apply Lemmas 2.3 and 2.2 to prove the non-degeneracy of any critical point. We proceed similarly to [6]. Differentiate $(2.2)$

$$
w_{x}^{\prime \prime}+\lambda f^{\prime}(u) w_{x}+\lambda f^{\prime \prime}(u) u_{x} w=0 .
$$


Multiplying the equation (2.27) by $w$, the equation (2.2) by $w_{x}$, integrating and subtracting, we express

$$
\lambda \int_{0}^{1} f^{\prime \prime}(u) u_{x} w^{2} d x=w^{\prime 2}(1)-\lambda w^{2}(0) f^{\prime}(u(0)) .
$$

Multiplying (2.1) by $u^{\prime}$, and integrating over $(0, x)$, we have

$$
{u^{\prime}}^{2}(x)=2 \lambda[F(u(0))-F(u(x))]=2 \lambda \int_{u(x)}^{u(0)} f(u) d u .
$$

Let as before $\xi \in(0,1)$ be the point where $u(\xi)=a$. Evaluating (2.29) at $x=\xi$, we see that $\int_{a}^{u(0)} f(u) d u>0$. Hence if the condition (2.25) is satisfied, we see that $u(0)>\tau$, and then $f^{\prime}(u(0))<0$. Hence the integral in $(2.28)$ is positive, and the lemma follows.

We now assume that (2.26) holds. Using the equations (2.1) and (2.2), it is easy to verify that (see e.g. [6])

$$
u^{\prime \prime}(x) w(x)-u^{\prime}(x) w^{\prime}(x)=-\lambda w(0) f(u(0)) \text { for all } x \in[-1,1] .
$$

Evaluating this expression at $x=1$, and using (2.29), we have

$$
w^{\prime 2}(1)=\frac{\lambda w^{2}(0) f^{2}(u(0))}{2 F(u(0))} .
$$

Using this in (2.28), we finally express

$$
\lambda \int_{0}^{1} f^{\prime \prime}(u) u_{x} w^{2} d x=\frac{w^{2}(0)}{2 F(\rho)} I(\rho),
$$

where we denote $\rho=u(0)$. By our assumption (2.26) $I(\beta) \geq 0$. Observe that

$$
I^{\prime}(\rho)=-2 F(\rho) f^{\prime \prime}(\rho) \geq 0 \text { for } \rho \geq \beta
$$

By Lemma 2.4, $\rho>\beta$, and hence $I(\rho)>0$, and the proof follows.

\section{Exact multiplicity of solutions for a class of equa- tions, generalizing cubic}

We can now easily describe the global picture.

Theorem 3.1 Consider the problem

$$
u^{\prime \prime}+\lambda f(u)=0 \quad x \in(-1,1), \quad u(-1)=u(1)=0 .
$$

Assume that the function $f(u)$ satisfies the conditions of Lemma 2.5, i.e. it satisfies the conditions (2.10) and (2.20), and either one of the conditions (2.25) and (2.26). 
Then there exists a critical $\lambda_{0}$, such that the problem (3.1) has exactly one positive solution for $0<\lambda<\lambda_{0}$, exactly two positive solutions at $\lambda=\lambda_{0}$, and exactly three positive solutions for $0<\lambda\rangle \lambda_{0}$. Moreover, all solutions lie on two smooth solution curves. One of the curves, referred to as the lower curve, starts at $(\lambda=0, u=0)$, it is increasing in $\lambda$, and $\lim _{\lambda \rightarrow \infty} u(x, \lambda)=a$. The upper curve is a parabola-like curve with exactly one turn to the right. (See Figure 1 below for the bifurcation diagram. We use $u(0)$, i.e. the $L^{\infty}$ norm, to identify the solution.)

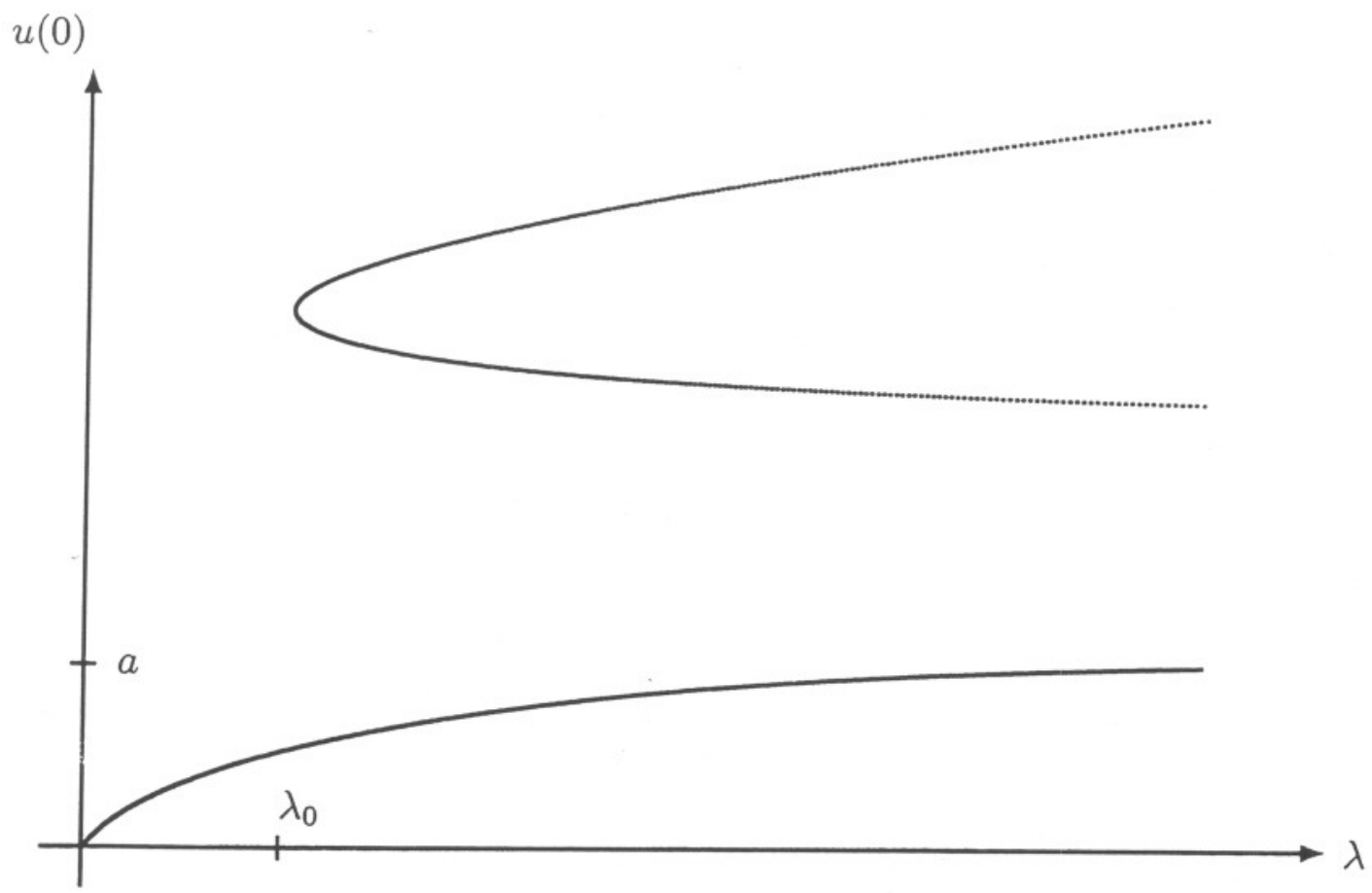

Figure 2

Proof. We begin by observing that there are exactly two solution curves, lower curve, where $u(0)<a$, and upper curve, where $u(0)>a$. Existence of these curves is well-known, see [6], so we only need to prove that there are no others. By an obvious modification of the Theorem 2.1 in P. Korman [5], as $\lambda$ tends to infinity, solutions on the lower curve tend to $a$ for all $x \in(-1,1)$, while on the upper curve one of the branches tends to $c$ for all $x \in(-1,1)$, and the other one tends to $a$ for $x \in(-1,1) \backslash\{0\}$. Define $\theta \in(b, c)$ by the condition $\int_{a}^{\theta} f(u) d u=0$, and a point $\xi \in(0,1)$, where $u(\xi)=a$. Multiplying the equation (3.1) by $u^{\prime}$, and integrating

$$
\frac{u^{\prime 2}(x)}{2}+\lambda \int_{a}^{u(x)} f(u) d u=\frac{1}{2}{u^{\prime}}^{2}(\xi) .
$$

It follows that $u(0)>\theta$ for any solution on the upper curve. As $\lambda \rightarrow \infty$, for solutions on the lower branch of the upper curve we have $u^{\prime}(\xi) \rightarrow 0$ (since solution is close to $a$ on most of the interval), and by (3.2) this implies $u(0) \rightarrow \theta$.

It is known, see [3], that the value of $u(0)$ uniquely identifies the solution $(\lambda, u(x))$. Since by the above remarks the upper and lower curves take up all 
possible values of $u(0)((0, a) \cup(\theta, c))$, it follows that there is "no room" for other curves of solutions.

The properties of the lower curve follow easily, since $f^{\prime}(u)<0$ for $u<a$. Turning to the upper curve, we need to show that it has exactly one turn. We consider a family of problems

$$
u^{\prime \prime}+\lambda g(u, \epsilon)=0 \quad x \in(-1,1), \quad u(-1)=u(1)=0,
$$

where $g(u, \epsilon)=f(u+\epsilon), 0 \leq \epsilon \leq a$. When $\epsilon=0$ we have the original problem (3.1). When $\epsilon=a$, we have $g(0, a)=0$, and $g(u, a)$ changes concavity exactly once. The solution curve is then a parabola-like curve, similar to the upper curve in the Figure 1, as was proved in [6], [8] and [10]. (Notice that at $\epsilon=a$ the lower curve disappears.)

Observe that either one of the conditions (2.25) and (2.26) holds for all $\epsilon \in[0, a]$. Indeed, assume first that (2.25) was true at $\epsilon=0$. What this condition says is that the integral of $f(u)$ from its first positive root to its point of global maximum is nonpositive. Clearly, the same remains true as we shift the graph to the left. Assume now that (2.26) was true at $\epsilon=0$. The point $\beta$ in that condition is the point where the straight line through the first positive root of $f(u)$ intersects the positive hump of $f(u)$. As we increase $\epsilon, \beta=\beta(\epsilon)$ moves to the left, however $f(\beta)$ and $f^{\prime}(\beta)>0$ remain unchanged, while $F(\beta)$ decreases. It follows that $I(\beta)$ increases in $\epsilon$, so the condition (2.26) holds for all $\epsilon \in[0, a]$.

Assume on the contrary that the upper curve has more than one turning point. By Lemmas 2.5 and 2.1 these turning points continue for all $\epsilon$, so that at $\epsilon=a$ we have more than one turning point on the upper curve, contradicting the previously mentioned results of [6], [8] and [10].

\section{Remarks}

1. Using Lemma 2.4, one can also simplify our original proof in [6].

2. More detailed information is available on the solutions, see [6]. In particular, solutions on the lower branch of the upper curve develop two transition layers on $(0,1)$, when $\lambda$ is large.

\section{References}

[1] I. Addou, Multiplicity results for classes of one-dimensional p-Laplacian boundaryvalue problems with cubic-like nonlinearities, Electron. J. Differential Equations No. $52,(2000)$.

[2] M.G. Crandall and P.H. Rabinowitz, Bifurcation, perturbation of simple eigenvalues and linearized stability, Arch. Rational Mech. Anal. 52 (1973) 161-180.

[3] E. N. Dancer, On the structure of solutions of an equation in catalysis theory when a parameter is large, J. Differential Equations 37 (1980), 404-437.

[4] B. Gidas, W.-M. Ni and L. Nirenberg, Symmetry and related properties via the maximum principle, Commun. Math. Phys. 68 (1979), 209-243. 
[5] P. Korman, Curves of sign-changing solutions for semilinear equations, Nonlinear Anal. TMA 51, no. 5 (2002), 801-820.

[6] P. Korman, Y. Li and T. Ouyang, Exact multiplicity results for boundary-value problems with nonlinearities generalising cubic, Proc. Royal Soc. Edinburgh, Ser. A 126A (1996), 599-616.

[7] P. Korman and T. Ouyang, Multiplicity results for two classes of boundary-value problems, SIAM J. Math. Anal. 26 (1995), 180-189.

[8] J. Smoller and A. Wasserman, Global bifurcation of steady-state solutions, J. Differential Equations 39 (1981), 269-290.

[9] J. Shi, Persistence and bifurcation of degenerate solutions, J. Funct. Anal. 169 (1999), 494-531.

[10] S.-H. Wang, A correction for a paper by J. Smoller and A. Wasserman, J. Differential Equations 77 (1989), 199-202.

[11] S.-H. Wang and N.D. Kazarinoff, Bifurcation of steady-state solutions for a scalar reaction-diffusion equation in one space variable, J. Austral. Math. Soc. Ser. A 52 (1992), 343-355. 